\title{
LETTER
}

Immunotherapy

\section{PMN-MDSC are a new target to rescue graft-versus-leukemia activity of NK cells in haplo-HSC transplantation}

\author{
Nicola Tumino ${ }^{1}$ - Francesca Besi ${ }^{1}$ - Anna Laura Di Pace ${ }^{1}$. Francesca Romana Mariotti ${ }^{1}$ Pietro Merli ${ }^{2}$. \\ Giuseppina Li Pira ${ }^{2}$ - Federica Galaverna ${ }^{2}$ - Angela Pitisci ${ }^{2}$ - Tiziano Ingegnere ${ }^{1}$ - Andrea Pelosi ${ }^{1} \cdot$ Linda Quatrini $^{1}$. \\ Enrico Munari $\mathbb{1}^{3,4} \cdot$ Franco Locatelli ${ }^{2,5} \cdot{\text { Lorenzo Moretta } \mathbb{I}^{1} \cdot \text { Paola Vacca }}^{1}$
}

Received: 23 May 2019 / Revised: 23 July 2019 / Accepted: 12 August 2019 / Published online: 4 October 2019

(c) The Author(s) 2019. This article is published with open access

\section{To the Editor:}

$\alpha \beta T$ cell- and B cell-depleted HLA-haploidentical haematopoietic stem cell transplantation (haplo-HSCT) is a lifesaving therapeutic option to treat patients with high-risk leukemia lacking an HLA-compatible donor [1]. In addition to hematopoietic stem cells (HSC), this manipulated graft contains mature donor-derived NK and $\gamma \delta \mathrm{T}$ cells, both exerting graft-versus-leukemia (GvL) activity and control of infections at early stages after transplantation [2]. Despite a satisfactory clinical outcome both in acute lymphoid and myeloid leukemia patients ( $70 \%$ probability of survival at 5 years), differently from the HSCT using "pure" CD34 precursors, the contribution of alloreactive NK cells (displaying KIR/HLA-I mismatch in the donor versus recipient direction), was found to be marginal [3, 4]. Notably, the G-CSF-induced mobilization of HSC in the donor causes relevant increases of different myeloid cells [5]. About 10\%

These authors contributed equally: Lorenzo Moretta, Paola Vacca

Supplementary information The online version of this article (https:// doi.org/10.1038/s41375-019-0585-7) contains supplementary material, which is available to authorized users.

Lorenzo Moretta

lorenzo.moretta@opbg.net

1 Immunology Research Area, IRCCS Bambino Gesù Pediatric Hospital, Rome, Italy

2 Department of Pediatric Hematology/Oncology, IRCCS Bambino Gesù Children's Hospital, Rome, Italy

3 Department of Pathology, Sacro Cuore Don Calabria, Negrar, VR, Italy

4 Department of Diagnostics and Public Health, University of Verona, Verona, Italy

5 Department of Pediatrics, Sapienza, University of Rome, Rome, Italy of donors, being "poor mobilizers", were further treated with plerixafor. However, no substantial differences were detected in cellular composition of the graft (data not shown). Since myeloid-derived suppressor cells (MDSC) could exert an inhibitory effect on NK- and $\gamma \delta \mathrm{T}$-cell effector function [6-9], it is crucial to assess whether such cells are present in the graft, possibly interfering with $\mathrm{GvL}$ activity. Human MDSC are classified in two major subsets: monocytic (Mo) $\left(\mathrm{CD}_{4} 5^{+} \mathrm{Lin}^{-} \mathrm{HLA}-\mathrm{DR}{ }^{-/{ }^{\prime l o w}} \mathrm{CD} 33^{+} \mathrm{CD} 11 \mathrm{~b}^{+}\right.$ $\left.\mathrm{CD} 14^{+} \mathrm{CD}_{66 \mathrm{~b}^{-}}\right)$and polymorphonuclear (PMN) $\left(\mathrm{CD} 45^{+}\right.$ $\mathrm{Lin}^{-} \mathrm{HLA}-\mathrm{DR}{ }^{-/{ }^{\prime l o w}} \mathrm{CD} 33^{+} \mathrm{CD} 11 \mathrm{~b}^{+} \mathrm{CD} 14^{-} \mathrm{CD}^{-} 6 \mathrm{~b}^{+}$), identified based on different surface antigens [6]. The expansion of these cell subsets was observed during pathological conditions, characterized by an high status of inflammation, such as viral/bacterial infections, autoimmune diseases, and tumors [10].

In this study, we show that PMN-MDSC are present in high proportions in the graft of $\alpha \beta$ T cell- and B cell-depleted transplants and exert a sharp inhibition on the effector functions of mature NK cells co-infused with the graft. A first set of experiments by flow-cytometry revealed that PMN-MDSC, but not Mo-MDSC, were highly enriched in mononuclear cell populations isolated from peripheral blood (PB) of G-CSF-mobilized haploidentical donors (Fig. 1a-c, Supplementary Materials and methods). Importantly, PMNMDSC were further enriched in the $\alpha \beta \mathrm{T}$ - and B-celldepleted grafts (in which they were $>10$-fold more than NK cells, data not shown). Of the 70 donors analyzed, $47 \%$ were male, median age $=40$ years and $53 \%$ female, median age $=37$ years (see Supplemental Materials and methods). Since mature PMN cells typically display a short life span ( $<24 \mathrm{~h}$ ) [11], we analyzed the in vitro survival of PMNMDSC isolated from mobilized donors. The percentage of viable cells decreased very slowly from day 1 to day 4 of culture, while it dropped sharply only after day 5 (Fig. 1d). These results indicate that donor-derived PMN-MDSC are characterized by a relatively prolonged life span, possibly 


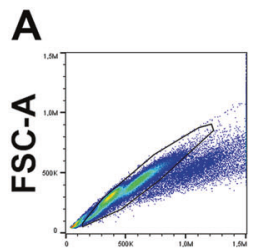

FSC-H

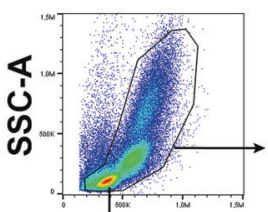

FSC-A

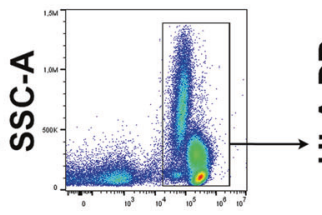

CD45

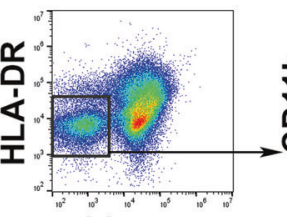

Lineage



CD33

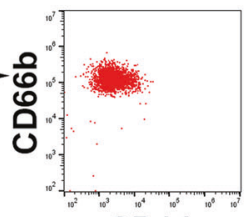

CD14

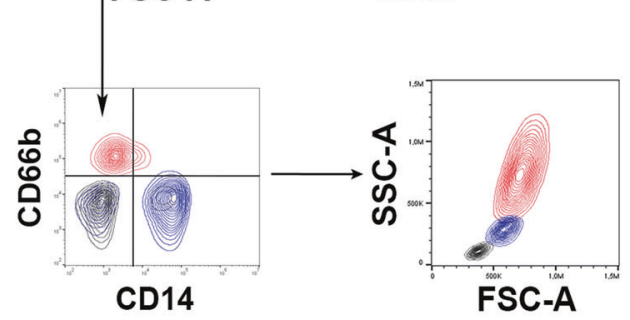

B

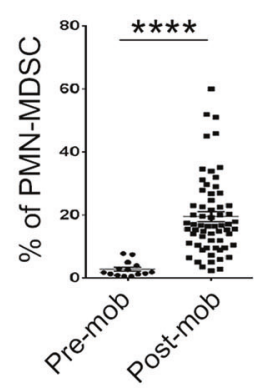

C

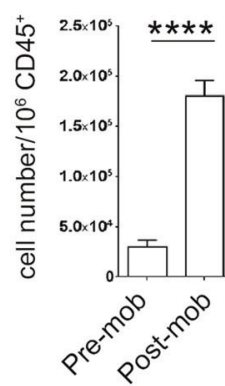

D

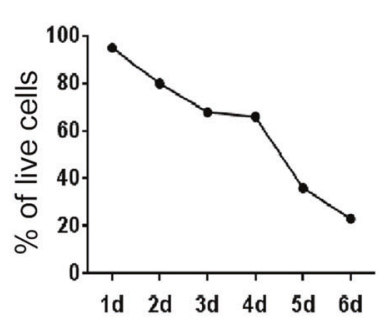

E

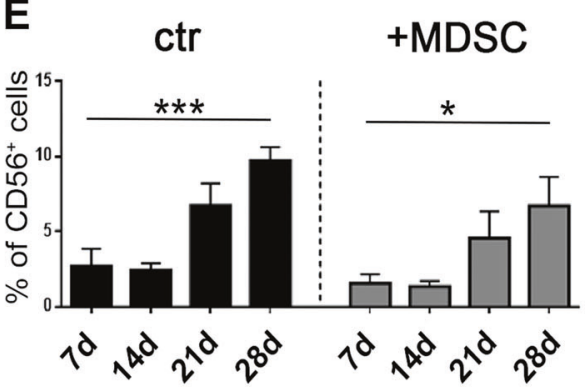

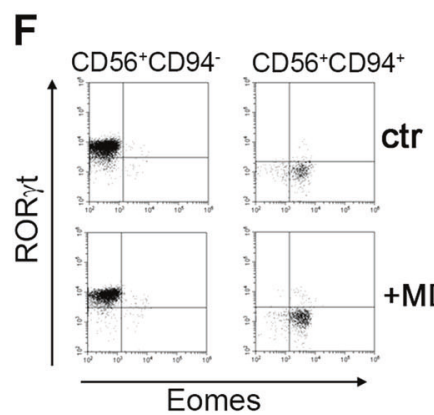

+ MDSC

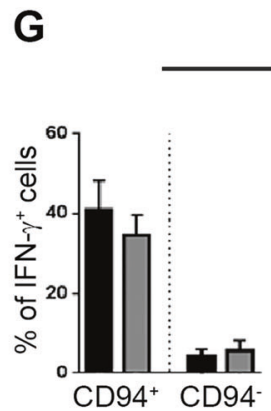

CD94+ CD94-

Fig. 1 Presence of PMN-MDSC in the apheresis of G-CSF-mobilized donors and their effect on NK/ILC differentiation from HSC. a-c Mononuclear cells present in the apheresis were analyzed by flowcytometry for the expression of specific markers allowing the identification of MDSC subsets. a Gating strategy adopted in one representative experiment out of 70 performed. b Percentages and c absolute numbers of PMN-MDSC $\left(\mathrm{CD}^{2} \mathrm{~b}^{+}\right.$cells) in different donors before (pre) and after (post) mobilization (mob). d Percentages of PMN-MDSC viable cells at different time points. e-g In these experiments, CD34 ${ }^{+}$HSC were isolated from the apheresis of G-CSFmobilized donors and cultured with cytokine-medium either in the absence (ctr) or in the presence (+MDSC) of PMN-MDSC

reflecting their immature stage and/or the treatment with GCSF. Given the high proportion of PMN-MDSC in the $\alpha \beta T$ cell- and B cell-depleted graft, first we asked whether they could compromise the differentiation of HSC towards NK cells. Figure 1e shows only a partial inhibitory effect on HSC differentiation towards $\mathrm{CD}^{+} 6^{+}$cells. We further

analyzed the composition and the functional capabilities of $\mathrm{CD}^{+} 6^{+}$progenies obtained in the presence of PMN-MDSC. As shown in Fig. 1f, both the EOMES-expressing $\mathrm{CD} 6^{+} \mathrm{CD} 94^{+}$, possibly corresponding to maturing $\mathrm{NK}$ cells, and the ROR $\gamma$ t-expressing $\mathrm{CD} 56^{+} \mathrm{CD} 94^{-}$, possibly corresponding to ILC3, were present. The two subsets could 
be unequivocally attributed to NK and ILC3, respectively, as revealed by the analysis of the expression of informative cytokines. Thus, CD94 ${ }^{+}$cells differentiated from both control and MDSC-containing cultures produced IFN- $\gamma$ and TNF- $\alpha$, but not IL-22 and IL-8. On the other hand, CD94produced both IL-22 and IL-8, as well as TNF- $\alpha$, but not IFN- $\gamma$ (Fig. 1g). These data indicate that the $\mathrm{CD}^{+} 4^{+}$and $\mathrm{CD}^{-} 4^{-}$cell subsets are both functional and their sets of cytokines indeed correspond to those typical of $\mathrm{NK}$ cells and ILC3, respectively. Taken together, these experiments indicate that PMN-MDSC could exert some inhibitory effect. However, the respective progenies display both phenotypic and functional characteristics of the two mature subsets.

An important improvement achieved with the $\alpha \beta T$ celland B cell-depleted haplo-HSCT, over the "pure" CD34 HSC, in the clinical outcome of high-risk leukemia patients is related to the co-infusion of mature donor NK and $\gamma \delta$ T cells [3]. Accordingly, another central question was whether PMN-MDSC, co-infused in the graft with NK and $\gamma \delta \mathrm{T}$ cells, were potentially capable of compromising the function and antileukemia activity of these effector cells. To this end, PMN-MDSC, freshly purified from apheresis of mobilized donors, were cultured for 48 $\mathrm{h}$ with allogenic, freshly isolated or IL-2-cultured NK cells at $1 / 1$ ratio. Figure $2 \mathrm{a}, \mathrm{b}$ shows a sharp inhibitory effect on the cytolytic activity (expressed as \% of killed target cells) of both fresh and IL-2-cultured NK cells against the NALM-18 leukemia cell line. Similarly, the cytokine production in both freshly isolated and IL-2cultured NK cells co-cultured with PMN-MDSC was strongly inhibited as compared with NK cells cultured alone (Fig. 2c, d). In addition, the expression of CD107a, a marker of cytolytic cell degranulation (and of cytolytic activity) was impaired (Fig. 2c, d). Similar results were obtained using as target cell the K562 erythroleukemia cell line (data not shown). In order to obtain a direct information on the effect of PMN-MDSC on donor NKcell-mediated killing of patient's leukemia blasts, experiments were performed using these blasts as target cells in a cytolytic assay. Cytolytic activity of donor NK cells co-cultured with PMN-MDSC was strongly inhibited (Fig. 2e). The PMN-MDSC inhibitory effect was confirmed also by the analysis of CD107a expression in donor NK cells upon interaction with leukemia blasts (Fig. 2f, g). These data further support the notion that, indeed, PMN-MDSC contained in the graft may exert an early inhibitory effect on NK cell-mediated GvL activity.

Since an inhibitory effect was detected also under transwell conditions (Fig. 2b), these data suggested the involvement of PMN-MDSC-derived soluble factors. Previous studies indicated that different inhibitory factors/ cytokines may affect the surface expression or the signaling capability of major activating NK receptors involved in tumor cell killing [12]. As shown in Fig. 2h, i, the expression of NKp46, NKp30, NKp44, CD16, NKG2D, DNAM-1, Granzyme B, and Perforin was significantly reduced when NK cells were co-cultured with PMNMDSC. In addition, the expression of the ITAM-bearing adapter polypeptides CD3 $\zeta$ and KARAP/DAP-12 mediating signal transduction of these activating receptors, was inhibited (Fig. 2i). Notably, the use of the IDO inhibitor $1 \mathrm{MT}$ and/or NS398 (PGE-2 inhibitor) could partially restore the NK cytolytic activity, thus indicating that IDO and PGE2 are involved in the suppression (Fig. 2j). Supplementary Fig. 1a, b shows the presence of IDO1 and IDO2 proteins and mRNA in PMN-MDSC. In contrast, neither nor-NOHA ( $L$-arginase inhibitor) nor anti-TGF- $\beta$ mAbs had any effect (data not shown).

Different physiologic and pathologic processes are induced/modulated by exosomes released from different cell types [13]. Thus, we assessed whether also PMNMDSC-derived exosomes contributed to the inhibitory effect on NK cell function. Exosomes were isolated from PMN-MDSC and the presence of exosome markers (CD63 and TSG101) documented by western blot (Fig. 2k). In contrast, the endoplasmic reticulum protein calnexin was present only in PMN-MDSC lysates, indicating that our purified preparations of exosomes were not contaminated by cellular components (Fig. 2k). Moreover, PMN-MDSCderived exosomes were internalized and clearly detectable in the cytoplasm of NK cells (Fig. 21-0). To assess their immunomodulatory potential, NK cells were cultured in the presence of PMN-MDSC-derived exosome. A significant impairment of NK-cell cytolytic activity was detected (Fig. 2p, q).

Our study indicates that PMN-MDSC derived from GCSF-mobilized donors can exert a potent inhibitory effect on the antileukemia activity of donor mature NK cells in peripheral blood stem cell transplant recipients. The inhibitory effect involves different mechanisms, including production of soluble factors and exosomes. Notably, in the $\alpha \beta \mathrm{T}$ cell- and B cell-depleted haplo-HSCT setting, the contribution of NK cell alloreactivity to prevent leukemia relapses was marginal, possibly reflecting a substantial impairment of the whole NK cell function [3]. No information exists on the degree of in vivo co-localization of grafted PMN-MDSC and NK cells. Of note, however, a strong inhibitory effect in vitro occurred also at $1 / 1$ ratio, while PMN-MDSC largely outnumber ( $>10$-fold) NK cells in the graft, thus rendering likely the occurrence of in vivo interactions and consequently of an inhibitory effect. Unlike NK cells, $\gamma \delta \mathrm{T}$ lymphocytes were mostly resistant to the inhibitory effect of PMN-MDSC (data not shown). Notably, patients given $\alpha \beta T$ cell- and B cell-depleted haplo-HSCT 

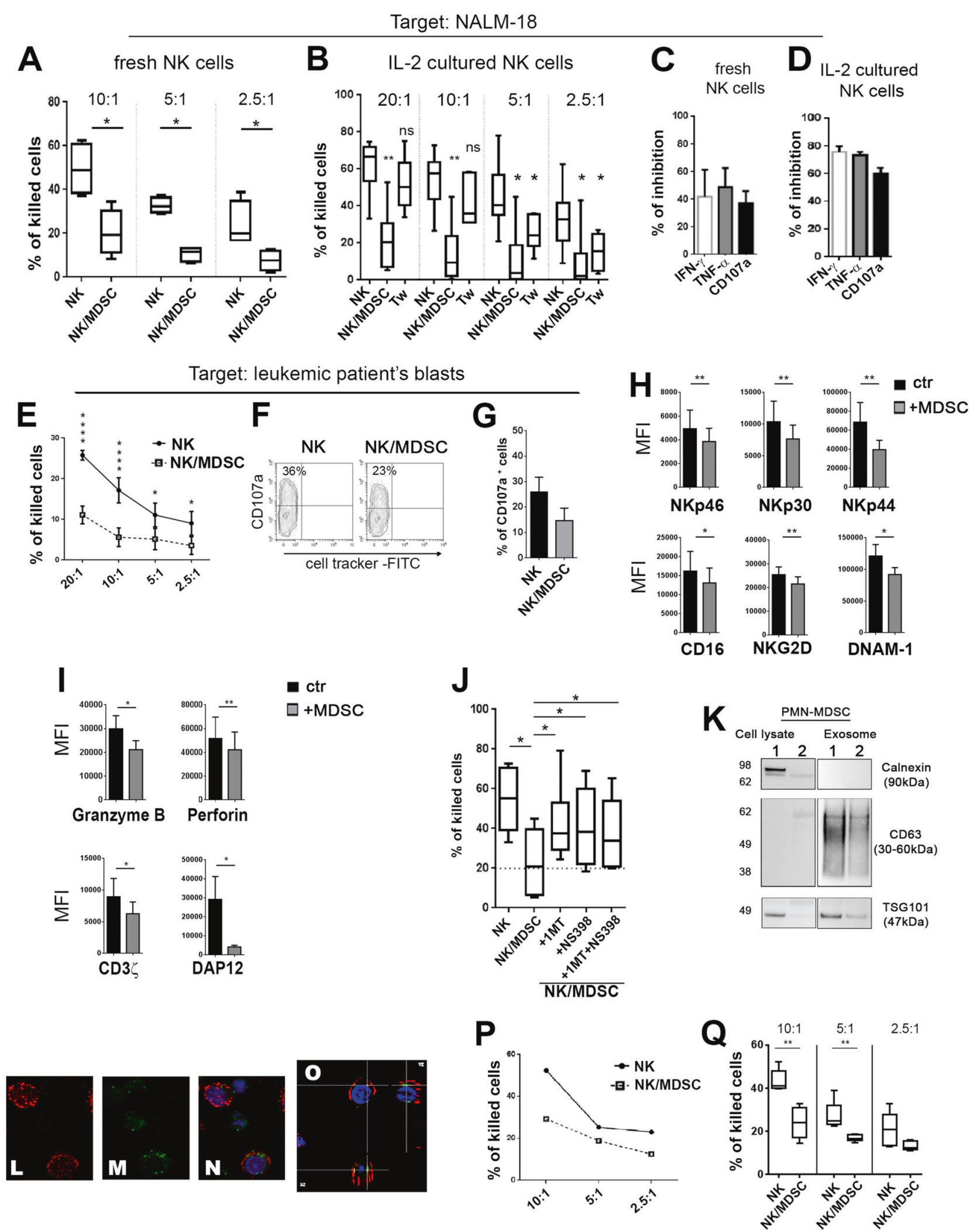

frequently received zoledronic acid that induces a marked $\gamma \delta \mathrm{T}$-cell expansion and also renders patient leukemia blasts more susceptible to the lytic activity of $\gamma \delta \mathrm{T}$ lymphocytes
[14, 15]. Our present data suggest an important approach (i.e., removal of PNM-MDSC from the graft) to rescue NK-cell function for further improving the efficacy of $\alpha \beta T$ 
Fig. 2 PMN-MDSC inhibit NK-cell function via IDO, PGE2, and exosomes. a-d In these experiments, the acute lymphoblastic leukemia cell line NALM-18 was used as target to asses either the NK-cell cytolytic activity or their cytokine production. Freshly isolated or IL-2expanded NK cells were cultured either alone (NK) or in the presence of PMN-MDSC at 1/1 ratio. After $48 \mathrm{~h}$ of co-culture, PMN-MDSC were removed by magnetic cell separation. The resulting NK cells were used as effector cells (indicated as NK/MDSC) in the cytolytic, cytokine and degranulation assays. Percentages of killed target cells by a freshly isolated $(n=4)$ or b IL-2-cultured NK cells $(n=6)$. b NK cells were cultured either alone or under cell-to-cell contact (NK/ MDSC) or under transwell (Tw) conditions as indicated in the figure. Cytokine production and degranulation of $\mathbf{c}$ freshly isolated $(n=3)$ or d IL-2 expanded NK cells $(n=5)$. Bars indicate the PMN-MDSCmediated inhibition $(\% \pm \mathrm{SEM})$, referred as the ratio between $\mathrm{NK} /$ MDSC co-cultures and NK cells cultured alone (arbitrarily normalized to 100). $\mathbf{e}-\mathbf{g}$ Evaluation of the effect of donor PMN-MDSC on autologous NK-cell cytotoxicity and degranulation capability (CD107a) against recipient leukemia blasts. e Percentages of killed patient's blasts by freshly isolated donor NK cells $(n=5)$. f One representative contour plots and $\mathbf{g}$ percentages \pm SEM of CD107a expression against patient's leukemia blasts $(n=3)$. In $\mathbf{h}-\mathbf{i}$ were analyzed the mechanism (s) involved in the NK-cell inhibition. IL-2-expanded NK cells were cultured either alone (ctr., black bars) or in the presence of PMNMDSC at $1 / 1$ ratio. After $48 \mathrm{~h}$ of co-culture, PMN-MDSC were removed by magnetic cell separation. The resulting NK cells (referred as +MDSC, gray bars) were analyzed. Mean fluorescence intensity (MFI) \pm SEM of the indicated (h) surface markers $(n=12)$, (i) granzyme B $(n=8)$, perforin $(n=9)$ and signaling molecules $\mathrm{CD} 3 \zeta(n=$ $9)$, and DAP12 $(n=6)$. j IL-2-expanded NK cells were cultured either alone or with PMN-MDSC (at 1/1 ratio) in the absence or in the presence of 1-MT (IDO inhibitor) and/or NS398 (PGE2 inhibitor). The cytolytic activity of NK cells cultured under different conditions was tested against NALM-18 cells $(n=6)$. Percentages \pm SEM of killed cells (E/T ratio used was 10/1). $\mathbf{k}$ Western blot analysis of calnexin, CD63, and TSG101 in PMN-MDSC cell lysates and in PMN-MDSCderived exosome. l-o Confocal microscopy of NK cells incubated for $48 \mathrm{~h}$ with exosomes purified from PMN-MDSC $\left(5 \mu \mathrm{g} / 5 \times 10^{5}\right.$ cells $)$. Confocal microscopic analysis of $1 \mathrm{CD}^{+} 6^{+} \mathrm{NK}$ cells (red), m PKH67 ${ }^{+}$-exosomes (green), $\mathbf{n}$ merge of $\mathrm{CD}^{+} 6^{+}$and exosome, nuclei in DAPI (blue); $\mathbf{o}$ confocal z-stack of merged section. Magnification $\times 60$. $\mathbf{o}, \mathbf{p}$ Percentages of killed NALM-18 cells after incubation with PMNMDSC-derived exosomes. $\mathbf{p}$ One representative experiment out of six performed and statistical analysis. A $p$ value $\leq 0.05$ was considered statistically significant. $* p \leq 0.05 ; * * p \leq 0.01 ; * * * p \leq 0.001 ; * * * p \leq$ $0.0001 ; \mathrm{ns}=$ not significant. Where not indicated, the data were not statistically significant

cell- and B cell-depleted haplo-HSCT. This additional graft manipulation step could have a further positive effect on the $\mathrm{GvL}$ activity considering that leukemia relapses still represent the main cause of mortality ( $>20 \%)$ [3]. In addition, it could ensure a more efficient viral protection, both these effects translating into a better patient's clinical outcome.

Acknowledgements This work was supported by grants from the Associazione Italiana per la Ricerca sul Cancro (AIRC)-Special Program Metastatic disease: the key unmet need in oncology 5 per mille 2018 Id. 21147 (LM and FL), AIRC IG 2017 Id. 19920 (LM), Ministero della Salute RF-2013, GR-2013-02356568 (PV) RC-2019 OPBG (LM, PV), Ministero dell'Istruzione, Università e Ricerca
(MIUR, grant PRIN 2017, FL). NT and ALDP are recipient of fellowships awarded by AIRC. The author thanks: V. D'Oria for technical support in Confocal microscopy (Confocal Microscopy Core Facility, IRCSS Bambino Gesù Children's Hospital, Rome, Italy), G. Cucci for secretary support (Immunology Research Area, IRCCS Bambino Gesù Pediatric Hospital, Rome, Italy).

Author contributions NT performed research, interpreted data, and wrote the paper; FB performed research and analyzed data; ALDP, FRM performed experiments; PM, GLP, FG provided samples and discussed data; TI, AP, LQ, EM, contributed to experiments and contributed to the final version of the paper; FL contributed patient samples, critically analyzed the experimental data and extensively reviewed the paper; LM, PV, designed and supervised the research and wrote the paper.

\section{Compliance with ethical standards}

Conflict of interest The authors declare that they have no conflict of interest.

Publisher's note Springer Nature remains neutral with regard to jurisdictional claims in published maps and institutional affiliations.

Open Access This article is licensed under a Creative Commons Attribution 4.0 International License, which permits use, sharing, adaptation, distribution and reproduction in any medium or format, as long as you give appropriate credit to the original author(s) and the source, provide a link to the Creative Commons license, and indicate if changes were made. The images or other third party material in this article are included in the article's Creative Commons license, unless indicated otherwise in a credit line to the material. If material is not included in the article's Creative Commons license and your intended use is not permitted by statutory regulation or exceeds the permitted use, you will need to obtain permission directly from the copyright holder. To view a copy of this license, visit http://creativecommons. org/licenses/by/4.0/.

\section{References}

1. Rocha V, Locatelli F. Searching for alternative hematopoietic stem cell donors for pediatric patients. Bone Marrow Transplant. 2008;41:207-14.

2. Locatelli F, Pende D, Falco M, Della Chiesa M, Moretta A, Moretta L. NK cells mediate a crucial graft-versus-leukemia effect in haploidentical-HSCT to cure high-risk acute leukemia. Trends Immunol. 2018;39:577-90.

3. Locatelli F, Merli P, Pagliara D, Li Pira G, Falco M, Pende D, et al. Outcome of children with acute leukemia given HLAhaploidentical HSCT after alphabeta T-cell and B-cell depletion. Blood. 2017;130:677-85.

4. Ruggeri L, Capanni M, Urbani E, Perruccio K, Shlomchik WD, Tosti A, et al. Effectiveness of donor natural killer cell alloreactivity in mismatched hematopoietic transplants. Science. 2002;295:2097-2100.

5. Luyckx A, Schouppe E, Rutgeerts O, Lenaerts C, Fevery S, Devos $\mathrm{T}$, et al. G-CSF stem cell mobilization in human donors induces polymorphonuclear and mononuclear myeloid-derived suppressor cells. Clin Immunol. 2012;143:83-87.

6. Bronte V, Brandau S, Chen SH, Colombo MP, Frey AB, Greten $\mathrm{TF}$, et al. Recommendations for myeloid-derived suppressor cell nomenclature and characterization standards. Nat Commun. 2016;7:12150. 
7. Poschke I, Kiessling R. On the armament and appearances of human myeloid-derived suppressor cells. Clin Immunol. 2012;144:250-68.

8. Pistoia V, Tumino N, Vacca P, Veneziani I, Moretta A, Locatelli F, et al. Human gammadelta T-cells: from surface receptors to the therapy of high-risk leukemias. Front Immunol. 2018;9:984.

9. Gabrilovich DI, Nagaraj S. Myeloid-derived suppressor cells as regulators of the immune system. Nat Rev Immunol. 2009;9: 162-74.

10. Veglia F, Perego M, Gabrilovich D. Myeloid-derived suppressor cells coming of age. Nat Immunol. 2018;19:108-19.

11. Scapini P, Cassatella MA. Social networking of human neutrophils within the immune system. Blood. 2014;124:710-9.
12. Vitale M, Cantoni C, Pietra G, Mingari MC, Moretta L. Effect of tumor cells and tumor microenvironment on NK-cell function. Eur J Immunol. 2014;44:1582-92.

13. Robbins PD, Morelli AE. Regulation of immune responses by extracellular vesicles. Nat Rev Immunol. 2014;14:195-208.

14. Bertaina A, Zorzoli A, Petretto A, Barbarito G, Inglese E, Merli P, et al. Zoledronic acid boosts gammadelta T-cell activity in children receiving alphabeta $(+) \mathrm{T}$ and $\mathrm{CD} 19(+)$ cell-depleted grafts from an HLA-haplo-identical donor. Oncoimmunology. 2017;6: e1216291.

15. Airoldi I, Bertaina A, Prigione I, Zorzoli A, Pagliara D, Cocco C, et al. gammadelta T-cell reconstitution after HLA-haploidentical hematopoietic transplantation depleted of TCR-alphabeta+/CD19+ lymphocytes. Blood. 2015;125:2349-58. 